\title{
A Quantum Wire Intersubband Emitter
}

Ingo Keck, Stefan Schmult, Werner Wegscheider

Institut für Experimentelle und Angewandte Physik,

Universität Regensburg, 93040 Regensburg, Germany

\author{
Martin Rother \\ Agfa-Gevaert AG, Abteilung CI-OE-RछD-IS, Tegernseer Landstr. 161, 81539 München, Germany
}

\author{
Andreas P. Mayer \\ Institut für Theoretische Physik, Universität Regensburg, 93040 Regensburg, Germany
}

\begin{abstract}
A quantum cascade emitter structure is suggested that is based on electronic transitions in an artificial band structure of coupled quantum wires in contrast to the conventional quantum layer systems. The electron transport is normal to the wires in a $\mathrm{GaAs} / \mathrm{Al}_{x} \mathrm{Ga}_{1-x}$ As heterostructure produced by the cleaved-edge overgrowth method.

For a realistic system of this type, the relevant rates of radiative transitions and of non-radiative transitions mediated by longitudinal optical phonons have been calculated and have been compared with corresponding data for quantum cascade emitters based on layered structures.
\end{abstract}

\section{INTRODUCTION}

Solid state lasers based on intersubband transitions in semiconductor heterostructures are a rapidly expanding field of research in view of a large variety of applications as compact mid- and far-infrared light sources. While the possibility of optical amplification by intersubband transitions in biased superlattices had already been predicted in 1971 [1], a successfully working quantum cascade laser (QCL) has first been reported in 1994 in the pioneering work of Faist at al. [2]. The basic principle of a QCL may be summarized as follows: In an active region, consisting of several quantum wells, an optical transition between two subbands, labeled (3) (upper) and (2) (lower), takes place. Population inversion between subbands (3) and (2) is achieved by a rapid non-radiative resonant transition, mediated by longitudinal optical (LO) phonons, from subband (2) to a lower subband (1). From (1), the electrons tunnel into the next active region, where they perform another optical transition. This concept has so far been put into practice using the subbands of semiconductor heterostructures in quantum well systems [2-4]. The dimensionality of the quantum confinement does not enter into the operating principle, so it may also be conceived for an array of quantum wires with the electrons being transported normal to the direction of the wires. In our case the quantum wires are produced by the cleaved edge overgrowth (CEO) technique [5]. An electrically pumped interband laser fabricated with this growth method has already been demonstrated [6].

The goal of this paper is to critically compare the advantages of a quantum wire system, fabricated by CEO, with the conventional systems containing (two-dimensional) quantum wells. For this purpose, transition rates have been calculated for both types of systems. The calculations have been done for realistic GaAs/ $\mathrm{Al}_{x} \mathrm{Ga}_{1-x} \mathrm{As}$ heterostructures. Systems of this type have been actually grown by molecular beam epitaxy. The electronic energy subbands of the conduction band near the center of the Brillouin zone and the envelope wavefunctions for these structures have been determined using a Schrödinger-Poisson solver [7].

The paper is organized in the following way: After a short introduction of the geometry of the quantum wire and quantum well structures investigated, the approximations used in the calculation of subbands and envelope functions are briefly described. Subsequently, results for optical and LO-phonon mediated non-radiative transition rates are presented and discussed. The paper ends with some concluding remarks.

\section{CHARACTERIZATION OF THE SYSTEM}

GaAs/AlGaAs heterostructures are investigated that are fabricated by the CEO method in the following way (Fig. 1):

In a first step, a layered $\mathrm{GaAs} / \mathrm{Al}_{0.33} \mathrm{Ga}_{0.67} \mathrm{As}$ quantum cascade structure - similar to that published in Ref. 3 without doping - is grown along the [001] crystal direction on a highly n-doped GaAs substrate, chosen for the later purpose of an n-contact. Subsequently, this structure is cleaved in-situ in the UHV of the growth chamber along the [110] crystal direction(cleavage plane: $\mathrm{x}-\mathrm{z}$ ). On the cleavage plane, a new set of layers is grown (growth direction: y). Following an $\mathrm{Al}_{0.33} \mathrm{Ga}_{0.67}$ As buffer layer of 5-10 nm thickness, an n-type modulation doping is situated with the purpose to establish the confinement potential along the second growth direction and to provide the electrons in the 
quantum wires. These wires form at the cleavage plane due to the deformation of the conduction band. They are extended along the z-direction. This structure is compared with conventional quantum cascade emitter geometries consisting of wells that are grown along the z-direction.

\section{SUBBAND STRUCTURE AND ENVELOPE FUNCTIONS}

For the heterostructures described in the previous section, the electronic subbands of the conduction band near the center of the Brillouin zone and the corresponding envelope wave functions have been determined with the help of the software package AQUILA [7] that solves self-consistently the Schrödinger equation

$$
\left\{-\nabla \cdot \frac{\hbar^{2}}{2 m^{*}(\mathbf{r})} \nabla+V_{C}(\mathbf{r})+\mathbf{F} \cdot \mathbf{r}+V_{H}(\mathbf{r})\right\} \psi_{j}(\mathbf{r})=E_{j} \psi_{j}(\mathbf{r})
$$

and the Poisson equation

$$
-\nabla \cdot\left\{\varepsilon(\mathbf{r}) \nabla V_{H}(\mathbf{r})\right\}=\rho(\mathbf{r})
$$

for a charge carrier distribution $\rho(\mathbf{r})$. Here, $m^{*}$ is the effective mass, $V_{C}$ the value of the lowest conduction band energy at the $\Gamma$ point of the Brillouin zone, $\mathbf{F}$ is an external bias field, $V_{H}$ is the Hartree potential, and $\varepsilon$ the local dielectric constant. The electron density $\rho$ is determined from the wave functions $\psi_{j}$ via

$$
\rho(\mathbf{r})=\sum_{j}\left|\psi_{j}(\mathbf{r})\right|^{2} f\left(E_{j}-E_{F}\right)
$$

with Fermi function $f$ and Fermi energy $E_{F}$. Coupling to the valence band is not taken into account.

For the quantum wire system described in the previous section, the envelope functions $\psi$ of the lowest conduction band $\left(\Gamma_{6}\right)$ are labeled by a subband index $\ell$ and a wavenumber $k_{3}$ :

$$
\psi_{k_{3}, \ell}(\mathbf{r})=\frac{1}{\sqrt{L}} e^{i k_{3} z} \chi_{\ell}\left(x, y \mid k_{3}\right)
$$

where $L$ is the length of the wires. The subband energies will be denoted by $E_{\ell}\left(k_{3}\right)$. In the quantum well structures, we decompose the wavefunctions as

$$
\psi_{k_{1}, k_{2}, \ell}(\mathbf{r})=\frac{1}{\sqrt{A}} e^{i\left(k_{1} x+k_{2} y\right)} \chi_{\ell}\left(z \mid k_{1}, k_{2}\right)
$$

and denote the subband energies by $E_{\ell}(\mathbf{k})$ with the two-component wavevector $\mathbf{k}=\left(k_{1}, k_{2}\right)$. ( $A$ is the area of the layers.) The functions $\chi$ are assumed to be normalized. In all our calculations of transition rates, the dependence of the functions $\chi$ on the wavenumber $k_{3}$ or (two-dimensional) wavevector $\mathbf{k}$ are neglected, and the dependence of the subband energies $E$ on $k_{3}$ or $\mathbf{k}$ are assumed to be approximately parabolic: $E_{\ell}\left(k_{3}\right)=\epsilon_{\ell}+\hbar^{2} k_{3}^{2} /\left(2 m_{\ell}^{*}\right)$, $E_{\ell}(\mathbf{k})=\epsilon_{\ell}+\hbar^{2}\left(k_{1}^{2}+k_{2}^{2}\right) /\left(2 m_{\ell}^{*}\right)$, with effective masses $m_{\ell}^{*}$. Equations (2.1) and (2.2) are solved numerically by discretization on a finite spatial domain. Because of translational invariance in the z-direction for the quantum wire system, this domain is two-dimensional and has been chosen to be rectangular. The following boundary conditions have been imposed at the boundaries of the domain:

(i) The wavefunction $\psi$ and the normal derivative of $\psi / m^{*}$ have to vanish.

(ii) The electric field has been required to vanish at the edge of the domain parallel to the x-axis far away from the cleavage plane, while at the edge near the cleavage plane, the electrostatic potential has been set equal to const. $+F x$. (The bias field is parallel to the $\mathrm{x}$-axis.)

(iii) The electrostatic potential is kept constant at the edges parallel to the y-axis.

The electrical boundary conditions (ii), (iii) are assumed to be a good approximation to the experimental situation. By adding an energy $F x$ to the calculated Fermi energy a spatially varying quasi-Fermi energy is introduced to prevent an accumulation of the charge carriers at the lowest energetic point of the structure.

The self-consistently determined potential $V_{S C}(x, y)=V_{C}(x, y)+V_{H}(x, y)$ is displayed in Fig. 2. A characteristic feature is the shallow tails of the troughs along the y-direction.

In Fig. 3 a-c, contour plots are shown of the electronic density $|\chi(x, y)|^{2}$ for the electronic states corresponding to the bottom of three subbands in the active region, labeled (I), (II), (III). The optical transition is supposed to occur between subbands (III) and (II), while the energy difference between subbands (II) and (I) has been arranged to be close to the energy of long-wavelength longitudinal optical phonons of bulk GaAs to have a high non-radiative 
transition rate and thus achieve optimal inversion of the population of the corresponding states in the subbands (II) and (III).

Because of the shallow potential troughs along the y-direction, a large number of additional subbands is found. These appear in series with the corresponding electron densities being localized in the same troughs, but exhibiting several maxima as one moves along the y-direction away from the cleavage plane. An example is given in Fig. $3 \mathrm{~d}$. The degree of localization decreases with increasing energy. When labeling the subbands $\ell=1,2,3, \ldots$ with increasing energy $\epsilon_{\ell}=E_{\ell}(0)$, subband (I) corresponds to $\ell=2$, (II) to $\ell=6$, (III) to $\ell=37$, and the state shown in Fig. 3 $\mathrm{d}$ corresponds to $\ell=25$. This is entirely different from the situation in the quantum well structures, which can be tailored such that there are only few subbands in the active region of coupled quantum wells. In order to recognize the quantum cascade emitter scheme, Fig. 3 e shows a cut through the four selected wave functions (Fig. 3 a - d) in the potential of Fig. 2 at $y=4950 \AA$. Additionally the Eigenenergies of the first 75 wave functions were plotted on the left side of Fig. 3 e.

To demonstrate the applicability of our numerical scheme to QCL systems, we have calculated transition energies $E\left(\ell \rightarrow \ell^{\prime}\right)=E_{\ell}(\mathbf{0})-E_{\ell^{\prime}}(\mathbf{0})$ for the two QCLs described in Refs. 3, 4 and compared them with the corresponding experimental and theoretical data published in these works. The results are shown in Table I. Depending on whether the calculations were carried out for a doped or an undoped system, we found two subbands with very small energy separation both contributing to transitions. In these cases, we have listed energy values that are averages over the two states. The overall small discrepancies may partly be attributed to the fact that our scheme does not account for non-parabolicity corrections of the conduction band. They may also be due to different material data used in the calculations.

When applying the bias field specified in Ref. $3(48 \mathrm{kV} / \mathrm{cm})$, the energy difference $E(2 \rightarrow 1)$ in our calculation was found to be smaller than the LO phonon energy $\hbar \omega_{0}$. Hence no population inversion is expected. Therefore, we have performed additional calculations for a bias field increased by a factor of 1.5, computationally found to be an applied electric field yielding $E(2 \rightarrow 1)>\hbar \omega_{0}$ and a large inversion ratio. In this way a critical comparison between layer and wire systems becomes possible. (However this does not mean that the device presented in Ref. 3 would not work under the specified bias of $48 \mathrm{kV} / \mathrm{cm}$.)

\section{OPTICAL TRANSITION RATES}

Within the dipole approximation, the rate for electronic transitions from subband $\ell$ to subband $\ell^{\prime}$, associated with spontaneous photon emission, is approximately given by

$$
\Gamma_{R}\left(\ell \rightarrow \ell^{\prime}\right) \approx \frac{e^{2} n}{\pi \hbar^{4} c^{3} \varepsilon_{0}}\left[\epsilon_{\ell}-\epsilon_{\ell^{\prime}}\right]^{3} D_{\ell \rightarrow \ell^{\prime}},
$$

and is independent of the subband wavevector to a good approximation, if the two parabolic (or paraboloidal) subbands have identical curvatures (i.e. the same effective mass). In the simple formula (3.1), an effective index of refraction $n$ has been attributed, and no waveguiding or cavity effects have been taken into account. $e$ is the elementary charge, $c$ the velocity of light in vacuum, and the factor $D_{\ell \rightarrow \ell^{\prime}}$ contains the dipole matrix elements $d_{x}^{\ell \rightarrow \ell^{\prime}}, d_{y}^{\ell \rightarrow \ell^{\prime}}$ or $d_{z}^{\ell \rightarrow \ell^{\prime}}$. For our system of quantum wires, $D_{\ell \rightarrow \ell^{\prime}}=\left|d_{x}^{\ell \rightarrow \ell^{\prime}}\right|^{2}+\left|d_{y}^{\ell \rightarrow \ell^{\prime}}\right|^{2}$, while in the quantum well systems $D_{\ell \rightarrow \ell^{\prime}}=\left|d_{z}^{\ell \rightarrow \ell^{\prime}}\right|^{2} / 3$, where

$$
\begin{gathered}
\left(\begin{array}{c}
d_{x}^{\ell \rightarrow \ell^{\prime}} \\
d_{y}^{\ell \rightarrow \ell^{\prime}}
\end{array}\right)=\int_{-\infty}^{\infty} d x \int_{-\infty}^{\infty} d y \chi_{\ell}^{*}(x, y)\left(\begin{array}{l}
x \\
y
\end{array}\right) \chi_{\ell^{\prime}}(x, y) \\
d_{z}^{\ell \rightarrow \ell^{\prime}}=\int_{-\infty}^{\infty} d z \chi_{\ell}^{*}(z) z \chi_{\ell^{\prime}}(z)
\end{gathered}
$$

(see also Refs. 8, 9).

The authors of Ref. 3 report a theoretical value for the dipole matrix element corresponding to the transition $3 \rightarrow 2$ in their system of $d_{z}^{\ell \rightarrow \ell^{\prime}}=1.6 \mathrm{~nm}$. For the same system, including doping as specified in Ref. 3 and a bias field of 72 $\mathrm{kV} / \mathrm{cm}$, we find a value of $d_{z}^{\ell \rightarrow \ell^{\prime}}=1.5 \mathrm{~nm}$. With a bias field of $48 \mathrm{kV} / \mathrm{cm}$ two almost degenerate energy levels were found, as already mentioned in section III. The average dipole matrix element becomes considerably smaller $(0.85 \mathrm{~nm}$ in the doped and $1.0 \mathrm{~nm}$ in the undoped case). The resulting averaged optical transition rates are $4.4 \mu \mathrm{s}^{-1}$ for the doped and $4.7 \mu \mathrm{s}^{-1}$ in the undoped case.

For the QCL system of Ref. 4, we find a dipole matrix element of $d_{z}^{3 \rightarrow 2}=2.0 \mathrm{~nm}$ in the undoped case very close to the theoretical value of $2.1 \mathrm{~nm}$ reported by the authors. When doping is included, the dipole matrix element is reduced to $d_{z}^{3 \rightarrow 2}=1.6 \mathrm{~nm}$. The corresponding transition rates are of the order of $11-13 \mu \mathrm{s}^{-1}$. 
These results for QCL systems based on quantum layers may now be compared to the corresponding data for our quantum wire system. Here, a dipole matrix element of $d_{x}^{\mathrm{III} \rightarrow \mathrm{II}}=1.45 \mathrm{~nm}$ between the subbands III and II is found. ( $\left(d_{y}^{\mathrm{III} \rightarrow \mathrm{II}} \mid\right.$ is negligible when compared to $\left|d_{x}^{\mathrm{III} \rightarrow \mathrm{II}}\right|$.) For the optical transition rate between these subbands, the calculation yields $\Gamma=29 \mu \mathrm{s}^{-1}$, which is considerably higher than the corresponding values for the two conventional QCL systems considered.

\section{NON-RADIATIVE TRANSITION RATES}

The most important mechanism that mediates non-radiative transitions between different subbands in QCL layered structures is scattering at longitudinal optic (LO) phonons, which couple to the carriers via their associated macroscopic electric field. To determine this macroscopic field, we treat the heterostructure as an effective homogeneous medium with plane-wave like phonon modes and use the LO phonon frequency $\omega_{0}$ of GaAs $\left(\hbar \omega_{0}=36.5\right.$ meV). This approximation is justified by the small aluminum content in the heterostructure. When treating structures with higher aluminum content, confined and interface phonons should be considered [10]. The bare electric potential associated with near zone center LO phonons is screened by the conduction electrons in the system. Following Smet et al. [8], we treat screening in a simple fashion by introducing a Thomas-Fermi wavenumber $q_{s}$. Accounting for the inhomogeneous distribution of carriers would substantially complicate the numerical evaluation. For a qualitative assessment of the effect of screening on the non-radiative transition rates, this approximation should be sufficient.

The Fourier transform of the total electric potential, $\Phi$, is then related to the normal coordinate of the LO phonon mode, $Q$, via

$$
\Phi(\mathbf{q})=i \omega_{0} \sqrt{8 \pi\left(\frac{1}{\varepsilon_{\infty}}-\frac{1}{\varepsilon_{0}}\right)} \frac{q}{q^{2}+q_{s}^{2}} Q(\mathbf{q}, \mathrm{LO}) .
$$

Using Fermi's Golden Rule, a formula is derived in a straightforward way for the transition rate $\Gamma_{\mathrm{LO}}\left(\mathbf{k} \ell \rightarrow \ell^{\prime}\right)$ of an electronic state with two-dimensional wavevector $\mathbf{k}$ in subband $\ell$ into the subband $\ell^{\prime}$ in a quantum layer system. It is in agreement with the expressions given in section III B 1 of Ref. 8 . If the transition occurs from the bottom of subband $\ell$, i.e. if $\mathbf{k}=\mathbf{0}$, this formula reduces to

$$
\begin{aligned}
\Gamma_{\mathrm{LO}}\left(\mathbf{0} \ell \rightarrow \ell^{\prime}\right)=C N_{ \pm} & \frac{1}{\sqrt{q_{ \pm}^{2}+q_{s}^{2}}} \int_{-\infty}^{\infty} d z \int_{-\infty}^{\infty} d z^{\prime}\left\{1-\frac{q_{s}^{2}}{2\left(q_{ \pm}^{2}+q_{s}^{2}\right)}+\frac{q_{s}^{2}\left|z-z^{\prime}\right|}{2\left(q_{ \pm}^{2}+q_{s}^{2}\right)}\right\} \\
& \times e^{-\sqrt{q_{ \pm}^{2}+q_{s}^{2}}\left|z-z^{\prime}\right|} \chi_{\ell}^{*}(z) \chi_{\ell^{\prime}}(z) \chi_{\ell}\left(z^{\prime}\right) \chi_{\ell^{\prime}}^{*}\left(z^{\prime}\right)
\end{aligned}
$$

(see also Ref. 11), where $N_{ \pm}=\left[\exp \left(\hbar \omega_{0} /\left(K_{B} T\right)\right)-1\right]^{-1}+(1 / 2) \pm(1 / 2)$ with $T$ being the lattice temperature, $q_{ \pm}=\sqrt{\left(2 m^{*} / \hbar^{2}\right)\left(\epsilon_{\ell}-\epsilon_{\ell^{\prime}} \mp \hbar \omega_{0}\right)}$, and we have neglected the difference of the effective masses of the two subbands, $m_{\ell}^{*}=m_{\ell^{\prime}}^{*}=m^{*}$. The upper sign refers to phonon emission, the lower to phonon absorption. The double integral on the right-hand side of (4.2) has been carried out numerically. The prefactor is given by $C=2 e^{2} m^{*} \omega_{0}\left(\varepsilon_{\infty}^{-1}-\varepsilon_{0}^{-1}\right) /\left(\pi \hbar^{2}\right)$, where $e$ is the elementary charge.

In the quantum wire system, the transition rate can be brought into the form

$$
\begin{gathered}
\Gamma_{\mathrm{LO}}\left(k_{3} \ell \rightarrow \ell^{\prime}\right)=C N_{ \pm} \int_{-\infty}^{\infty} d x \int_{-\infty}^{\infty} d y \int_{-\infty}^{\infty} d x^{\prime} \int_{-\infty}^{\infty} d y^{\prime} \sum_{j=1}^{2} \frac{1}{\left|k_{3}-\kappa_{\mp}^{(j)}\right|} \\
\times\left\{K_{0}\left(\rho \sqrt{\kappa_{\mp}^{(j) 2}+q_{s}^{2}}\right)-\frac{\rho q_{s}^{2}}{2 \sqrt{\kappa_{\mp}^{(j) 2}+q_{s}^{2}}} K_{1}\left(\rho \sqrt{\kappa_{\mp}^{(j) 2}+q_{s}^{2}}\right)\right\} \chi_{\ell}^{*}(x, y) \chi_{\ell^{\prime}}(x, y) \chi_{\ell}\left(x^{\prime}, y^{\prime}\right) \chi_{\ell^{\prime}}^{*}\left(x^{\prime}, y^{\prime}\right) .
\end{gathered}
$$

In (4.3), we have introduced the auxiliary variables $\rho=\sqrt{\left(x-x^{\prime}\right)^{2}+\left(y-y^{\prime}\right)^{2}}, \quad \kappa_{\mp}^{(1)}=k_{3}+$ $\sqrt{k_{3}^{2}+2 m^{*}\left(\epsilon_{\ell}-\epsilon_{\ell^{\prime}} \mp \hbar \omega_{0}\right) / \hbar^{2}}$, and $\kappa_{\mp}^{(2)}=k_{3}-\sqrt{k_{3}^{2}+2 m^{*}\left(\epsilon_{\ell}-\epsilon_{\ell^{\prime}} \mp \hbar \omega_{0}\right) / \hbar^{2}} . \quad K_{0}$ and $K_{1}$ are Macdonald functions of order 0 and 1, respectively. The four-fold integral on the right-hand side of (4.3) has been carried out numerically, and care had to be taken because of the logarithmic singularity of the integrand at $\rho=0$. 
An important qualitative difference between the LO-phonon mediated transition rates in ideal quantum layer and ideal quantum wire systems is the fact that in the latter case, $\Gamma_{\mathrm{LO}}\left(k_{3} \ell \rightarrow \ell^{\prime}\right)$ diverges if the resonance condition

$$
\epsilon_{\ell}+\left(\hbar k_{3}\right)^{2} /\left(2 m^{*}\right)=\epsilon_{\ell^{\prime}} \pm \hbar \omega_{0}
$$

is met $[12-18]$, i.e. if the transition is into the bottom of the subband $\ell^{\prime}$, while $\Gamma_{\mathrm{LO}}\left(\mathbf{k} \ell \rightarrow \ell^{\prime}\right)$, the transition rate for layered systems, remains finite even in the absence of screening. For the special case $\mathbf{k}=\mathbf{0}$ (equation (4.2)), this is easily verified. If $q_{s}=0$, one has to make use of the orthogonality of the functions $\chi_{\ell}$ and $\chi_{\ell^{\prime}}$ and expand the exponential $\exp \left(-q_{\perp}\left|z-z^{\prime}\right|\right)$ in powers of its argument. The finiteness of $\Gamma_{\mathrm{LO}}\left(\mathbf{k} \ell \rightarrow \ell^{\prime}\right)$ can also be shown for $\mathbf{k} \neq \mathbf{0}$.

The divergence of $\Gamma_{L O}$ for the quantum wire systems at the resonance condition (4.4) implies that by a proper design of the quantum wire structure, the non-radiative transition rate for an electronic state with wavenumber $k_{3}$ in the $\ell$-th subband may ideally be made arbitrarily large. In a real system a number of other effects will lead to a finite lifetime of this state [15], especially spatial inhomogeneities of the structure, but these can be controlled experimentally to a high degree. Therefore, the population inversion of the subbands (III) and (II) in the active region of a QCL system based on quantum wires can be made very high.

Table II shows numerical results for LO phonon-mediated transition rates of the quantum wire system compared to corresponding data for the two layered systems of Refs. 3, 4. The rates listed in this table always refer to electronic transitions from the bottom of a subband into another subband. (In the cases of two energetically close subbands representing the upper laser state the given transition rates in Table II are averages over the two initial subbands and sums over the two final subbands.) The transition from level (II) to level (I) in the quantum wire system is found to be faster by a factor of 7 as compared to the transition rate $(2) \rightarrow(1)$ in the layered system specified in Ref. 3 or approximately a factor of 3 in comparison to our calculations for the layered structures. The results listed in Table II have been obtained with the approximations outlined above. To achieve precise values for the transition rates near the resonance, even in the absence of spatial inhomogeneities and other effects not considered here, the detailed phonon spectrum of the heterostructure would have to be known including phonon modes localized at interfaces. However, this will not change the general conclusion that high inversion factors can be reached in the quantum wire case.

In the layered systems with three confined subbands per active region, the inversion factor is usually estimated as $N_{3} / N_{2}=\Gamma_{\mathrm{LO}}(2 \rightarrow 1) / \Gamma_{\mathrm{LO}}(3 \rightarrow 2)$, where $N_{j}$ is the population number of the $j$-th subband. Values of the order of 8 - 16 have been reached. To obtain a rough estimate for the inversion factor $N_{\text {III }} / N_{\text {II }}$ of our quantum wire system, we make the following assumptions:

i) The injector populates subband 37 (=III) only.

ii) The intrasubband relaxation is sufficiently fast that the carriers may be assumed to relax instantaneously to the bottom of the subbands.

iii) Further relaxation mechanisms can be neglected.

With these simplifying assumptions, an inversion factor $N_{\mathrm{III}} / N_{\mathrm{II}}=163$ results, while $\sum_{\ell^{\prime}} \Gamma_{\mathrm{LO}}\left(I I \rightarrow \ell^{\prime}\right) / \Gamma_{\mathrm{LO}}(I I I \rightarrow$ $I I)=226$ gives a higher value.

Clearly, assumptions (ii) and (iii) are difficult to justify. While the results of Harrison [19] suggest that (ii) is satisfied for certain layered structures due to electron-electron scattering, this need not be the case for wire systems with many subbands having small energy separation. However, because of lack of quantitative data for the intraand inter-subband relaxation due to electron-electron interaction, the above value may be regarded as a first rough estimate.

With increasing carrier concentration, the screening reduces the rate of non-radiative transitions due to the macroscopic field associated with LO-phonons. In Fig. 4 the dependence on the screening wavenumber $q_{s}$ of the transition rates between subbands 2 and 1 in the QCL structure of Sirtori et al. [3] and of our quantum wire structure is shown. For sufficiently high carrier concentrations (i.e. sufficiently high values of $q_{s}$ ), the transition rate depends strongly on $q_{s}$, and the population inversion will eventually be suppressed in the layered structure.

Fig. 4 also demonstrates the divergence of $\Gamma_{L O}$ in the ideal quantum wire system, while $\Gamma_{L O}$ reaches a finite limiting value as $E(2 \rightarrow 1) \rightarrow \hbar \omega_{0}$ in the layered structure. Hence, it should be possible to maintain population inversion in the wire structures even at high carrier concentrations.

Apart from the population inversion, it is the achievable optical gain that is relevant for laser application of our heterostructure. This quantity strongly depends on the total non-radiative decay rate of the upper level involved in the optical transition ((III) for wire systems, (3) for layer systems). The data displayed in Table II show that the decay rates from the bottom of subband (III) into subbands (I) and (II) are much smaller than the corresponding rates from subband (3) into subbands (1) and (2) in the layered systems. This finding may be no surprise when considering the dimensional reduction of the number of final states in the subbands of the wire system as compared to the layered systems. However, the total non-radiative decay rate of electrons at the bottom of subband (III) into lower subbands $\left(\approx 1.1 \mathrm{ps}^{-1}\right)$ turns out to be of the same order of magnitude as the corresponding values of subband (3) in the quantum layer systems $\left(0.6-0.7 \mathrm{ps}^{-1}\right.$ for the QCL system of Ref. 3 and $0.8-1.8 \mathrm{ps}^{-1}$ for the system of Ref. 4). 
In our determination of the total non-radiative decay rate in the wire system, we have summed over all transitions to lower subbands $\ell^{\prime}$. The decay rates $\Gamma_{L O}\left(0 \mathrm{III} \rightarrow \ell^{\prime}\right)$ are presented graphically in Fig. 5 .

The large difference between the transition rate $\Gamma_{L O}(0 \mathrm{III} \rightarrow \mathrm{II})+\Gamma_{L O}(0 \mathrm{III} \rightarrow \mathrm{I})$ and the total decay rate $\sum_{\ell^{\prime}} \Gamma_{L O}\left(0 \mathrm{III} \rightarrow \ell^{\prime}\right)$ is due to the many additional subbands present in the wire structure. Here, the strong enhancement of the non-radiative decay near the resonance condition (4.4) is a disadvantage, as transitions into subbands other than (I) and (II) can be very efficient if the energy separation is close to $\hbar \omega_{0}$. Also, there can be enhanced loss due to LO phonon absorption, i.e. transitions into higher subbands, at elevated temperatures. (The results of Table II refer to $T=77 \mathrm{~K}$.)

\section{CONCLUSION}

In summary, an intersubband emitter system is suggested that is based on realistic quantum wires which can be fabricated by the cleaved edge overgrowth technique. Optical and non-radiative LO phonon mediated transition rates have been calculated for this system using the effective mass approximation with envelope functions determined within the Hartree approximation. The results were compared with the corresponding data for two quantum cascade laser structures consisting of multiple wells. The quantum wire system has been found superior over the layered structures in the optical transition rate and especially in the possibility to reach a high degree of population inversion. When considering the non-radiative decay rate of the upper level in the optical transition, which is an important loss mechanism and is supposed to play an essential role for the gain in laser applications, the quantum wire structure analyzed in this paper is not superior over the layered systems. This is due to the existence of a large number of subbands in the active region of the emitter. However, it may well be possible to further optimize the heterostructure moving energy differences out of resonance with LO phonons to reduce the efficiency of these additional decay channels.

After this first study of a realistic candidate system for an intersubband emitter based on quantum wires, further work is needed to achieve more quantitative results for the relevant transition rates, which should explicitly account for electron-electron scattering and coupling to acoustic phonons, and a detailed theory for electronic transport in this type of system has to be worked out that includes electron injection. Especially, relaxation via electron-electron scattering has to be analyzed in detail along the lines of Refs. 8, 20, 21 for layered systems and Ref. 22 in the quantum wire case. The increasing importance of electron-electron scattering for small subband separations has been stressed by Kinsler et al. [21], and it may play a dominant role even for inter-subband relaxation in our quantum wire system that exhibits many subbands with small energy separation. For a quantitative assessment of electron-electron relaxation processes in quantum cascade emitter devices, knowledge about the electron distribution function would be required, that may be gained in transport calculations similar to those reported in Refs. 23, 24, where the LO-phonon mediated transition rates enter as relevant parameters.

\section{ACKNOWLEDGMENT}

It is a pleasure to thank A. Wacker for a helpful discussion. Financial support from the Deutsche Forschungsgemeinschaft (Graduiertenkolleg "Nonlinearity and Non-Equilibrium in Condensed Matter") is gratefully acknowledged.

[1] R.F. Kazarinov and R.A. Suris, Sov. Phys. Semicond. 5, 707 (1971).

[2] J. Faist, F. Capasso, D.L. Sivco, C. Sirtori, A.L. Hutchinson, and A.Y. Cho, Science 264, 553 (1994).

[3] C. Sirtori, P. Kruck, S. Barbieri, P. Collot, J. Nagle, M. Beck, J. Faist, and U. Oesterle, Appl. Phys. Lett. 73, 3486 (1998).

[4] P. Kruck, H. Page, C. Sirtori, S. Barbieri, M. Stellmacher, and J. Nagle, Appl. Phys. Lett. 76, 3340 (2000).

[5] L. Pfeiffer, H.L. Störmer, K.W. West, A.R. Goñi, A. Pinzcuk, R.C. Ashoori, M.M. Dignam and W. Wegscheider, J. Crystal Growth 127, 84957 (1993).

[6] W. Wegscheider, L. Pfeiffer, K. West, and R.E. Leibenguth, Appl. Phys. Lett. 65, 2510 (1994).

[7] M. Rother, PhD Dissertation, Technical University of Munich, 1999.

[8] J.H. Smet, C.G. Fonstad, and Q. Hu, J. Appl. Phys. 79, 9305 (1996).

[9] S. Briggs, D. Jovanovic, and J.P. Leburton, Appl. Phys. Lett. 54, 2012 (1989).

[10] C. Becker, C. Sirtori, H. Page, A. Robertson, V. Ortiz, and X. Marcadet, Phys. Rev. B 65, 085305 (2002).

[11] R. Ferreira and G. Bastard, Phys. Rev. B 40, 1074 (1989).

[12] J.P. Leburton, J. Appl. Phys. 56, 2850 (1984).

[13] N.C. Constantinou and B.K. Ridley, J. Phys.: Condens. Matter 1, 2283 (1989).

[14] U. Bockelmann and G. Bastard, Phys. Rev. B 42, 8947 (1990). 
TABLE I: Transition energies $E\left(\ell \rightarrow \ell^{\prime}\right)$ in meV for the QCL systems of Ref. 3 (A), Ref. 4 (B), and the quantum wire system considered here $(\mathrm{C})$.

\begin{tabular}{|c|c|c|c|c|c|c|}
\hline A & $\begin{array}{l}3 \rightarrow 2 \\
2 \rightarrow 1 \\
\end{array}$ & $\begin{array}{c}153.3^{a} \\
33.5^{a} \\
\end{array}$ & $\begin{array}{c}154.8^{b} \\
33.1^{b} \\
\end{array}$ & $\begin{array}{c}163.3^{c} \\
37.9^{c}\end{array}$ & $\begin{array}{c}134^{d} \\
38^{d}\end{array}$ & $\begin{array}{c}131.6^{e} \\
35^{e} \\
\end{array}$ \\
\hline \multirow[t]{2}{*}{ B } & $3 \rightarrow 2$ & $126.4^{a}$ & $125.4^{b}$ & & $112^{d}$ & $110.5^{e}$ \\
\hline & $2 \rightarrow 1$ & $39.5^{a}$ & $40.0^{b}$ & & $\approx \hbar \omega_{0}{ }^{d}$ & $37^{e}$ \\
\hline$C$ & $37 \rightarrow 6$ & 150.9 & & & & \\
\hline & $6 \rightarrow 2$ & 37.3 & & & & \\
\hline
\end{tabular}

${ }^{a}$ With bias field specified by the authors, no doping.

${ }^{b}$ With bias field and doping specified by the authors.

${ }^{c}$ With doping specified by the authors and bias field $72 \mathrm{kV} / \mathrm{cm}$.

${ }^{d}$ Theoretical value given by the authors.

${ }^{e}$ Experimental value given by the authors.

TABLE II: Non-radiative transition rates mediated by LO phonons, in $\mathrm{ps}^{-1}$, for the QCL systems of Ref. 3 (A), Ref. 4 (B), and the quantum wire system considered here $(\mathrm{C})$. Lattice temperature: $T=77 \mathrm{~K}$.

\begin{tabular}{|c|c|c|c|c|c|}
\hline \multirow[t]{3}{*}{ A } & $3 \rightarrow 2$ & $0.207^{a}$ & $0.203^{b}$ & $0.466^{c}$ & $0.417^{d}$ \\
\hline & $3 \rightarrow 1$ & $0.143^{a}$ & $0.149^{b}$ & $0.251^{c}$ & $0.250^{d}$ \\
\hline & $2 \rightarrow 1$ & & & $8.15^{c}$ & $3.33^{d}$ \\
\hline \multirow[t]{3}{*}{ B } & $3 \rightarrow 2$ & $0.937^{a}$ & $1.124^{b}$ & & $0.5^{d}$ \\
\hline & $3 \rightarrow 1$ & $0.597^{a}$ & $0.685^{b}$ & & $0.33^{d}$ \\
\hline & $2 \rightarrow 1$ & $8.19^{a}$ & $6.74^{b}$ & & \\
\hline \multirow[t]{4}{*}{$\mathrm{C}$} & $37 \rightarrow 6$ & 0.11 & & & \\
\hline & $37 \rightarrow 2$ & 0.08 & & & \\
\hline & $6 \rightarrow 2$ & 24 & & & \\
\hline & $\ell^{\prime} 37 \rightarrow$ & $\approx 1.1$ & & & \\
\hline
\end{tabular}

${ }^{a}$ With bias field specified by the authors, no doping.

${ }^{b}$ With bias field and doping specified by the authors.

${ }^{c}$ With doping specified by the authors and bias field $72 \mathrm{kV} / \mathrm{cm}$.

${ }^{d}$ Theoretical value given by the authors.

[15] R. Mickevicius, V.V. Mitin, K.W. Kim, M.A. Stroscio, and G.J. Jafrate, J. Phys.: Condens. Matter 4, 4959 (1992).

[16] P.A. Knipp and T.L. Reinecke, Phys. Rev. B 48, 5700 (1993).

[17] B. Tanatar, Phys. Rev. B 48, 12001 (1993).

[18] S. Das Sarma and V.B. Campos, Phys. Rev. B 49, 1867 (1994).

19] P. Harrison, Appl. Phys. Lett. 75, 2800 (1999).

[20] S.M. Goodnick and P. Lugli, Phys. Rev. B 37, 2578 (1988).

[21] P. Kinsler, P. Harrison, and R.W. Kelsall, Phys. Rev. B 58, 4771 (1998).

[22] M.R.S. Tavares, S. Das Sarma, and G.-Q. Hai, Phys. Rev. B 63, 045324 (2001).

[23] R.C. Iotti and F. Rossi, Appl. Phys. Lett. 76, 2265 (2000).

[24] R.C. Iotti and F. Rossi, Phys. Rev. Lett. 87, 146603 (2001). 


\section{Figures}

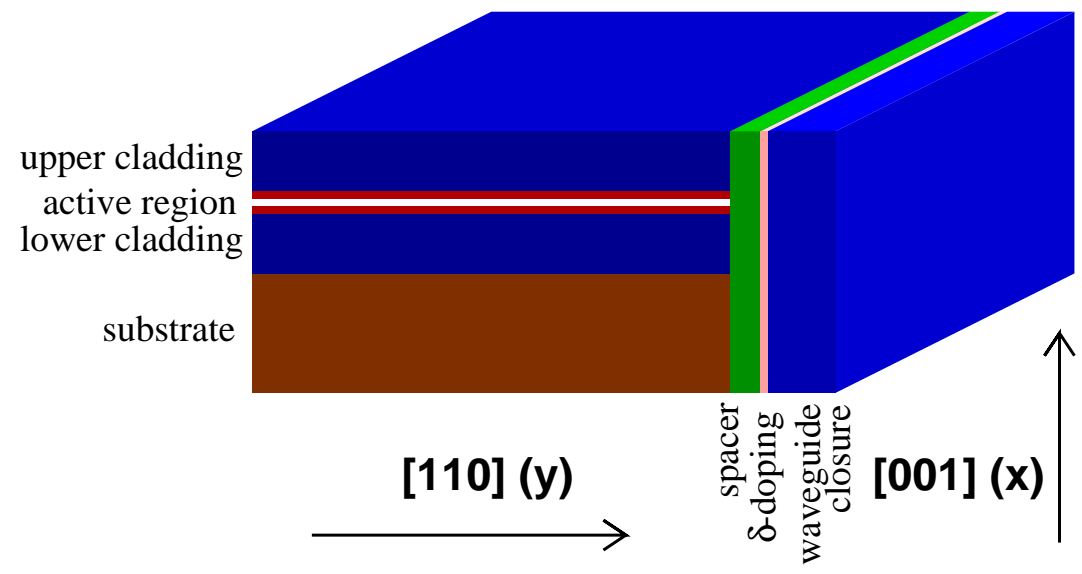

Fig. 1. Heterostructure analyzed in this work.

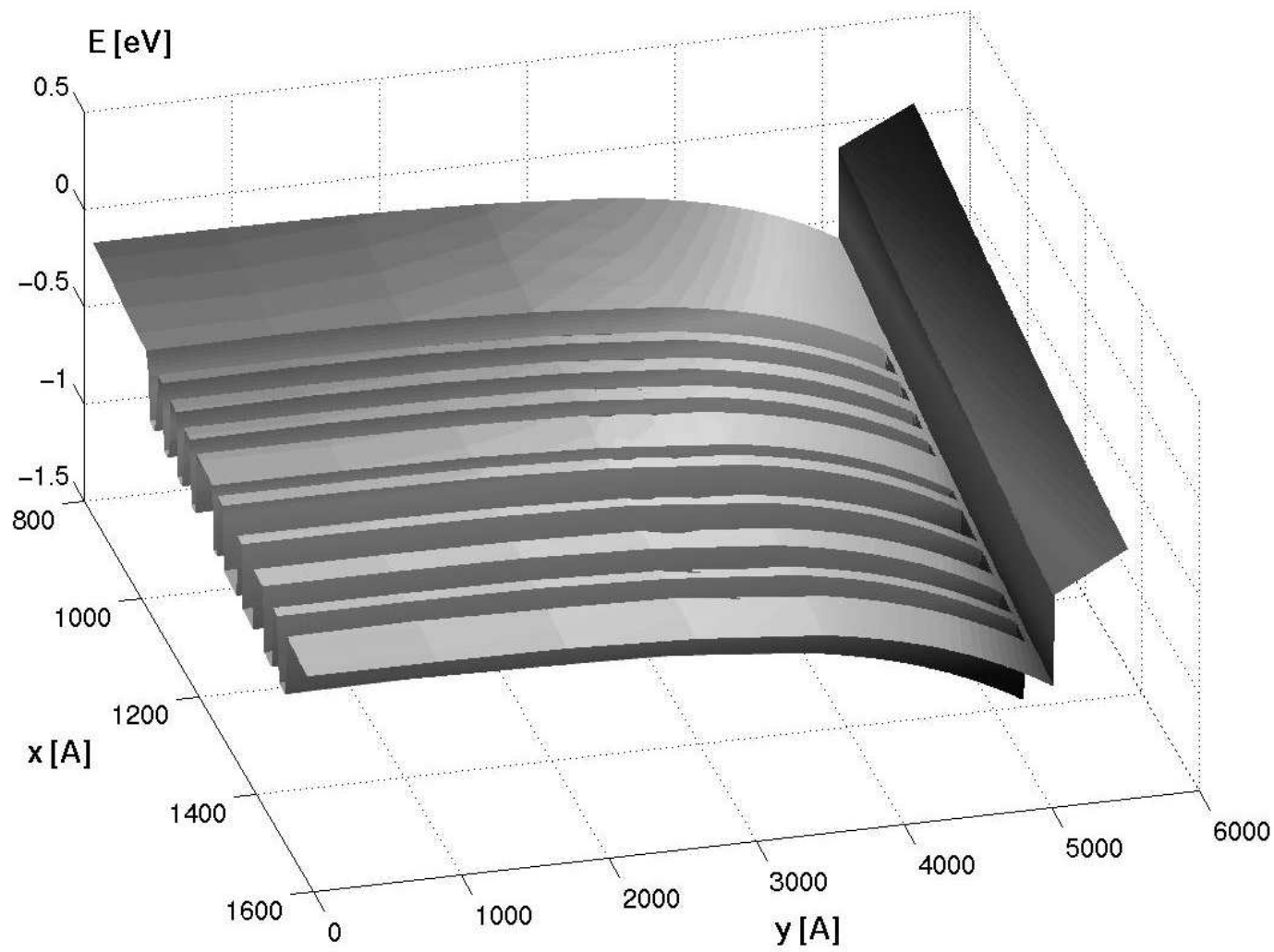

Fig. 2. Self-consistent potential $V_{S C}$ of the active region in the structure of Fig. 1. 


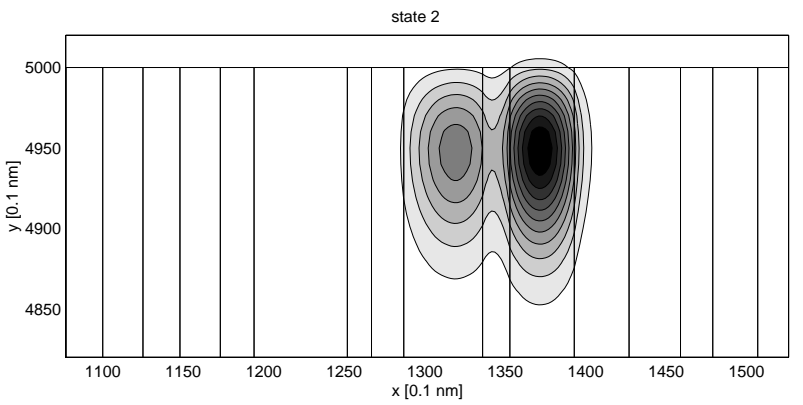

(a)

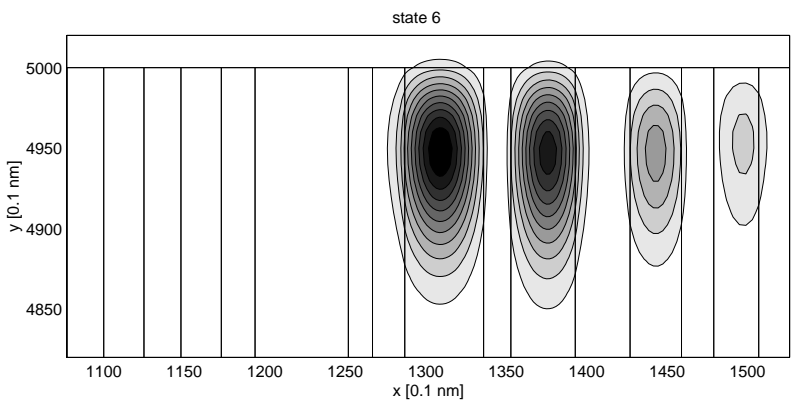

(b)

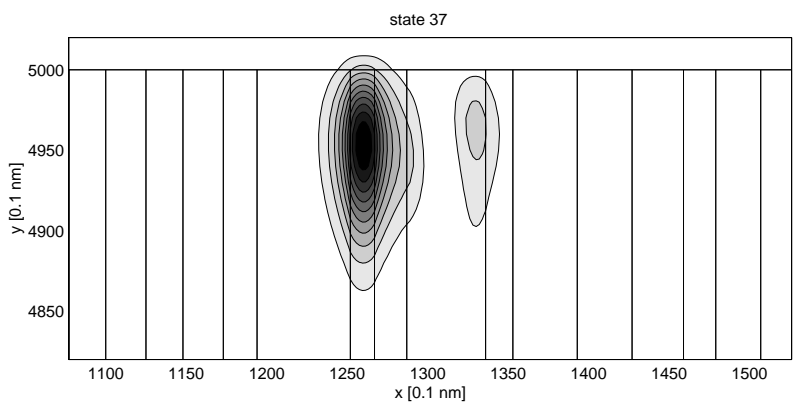

(c)

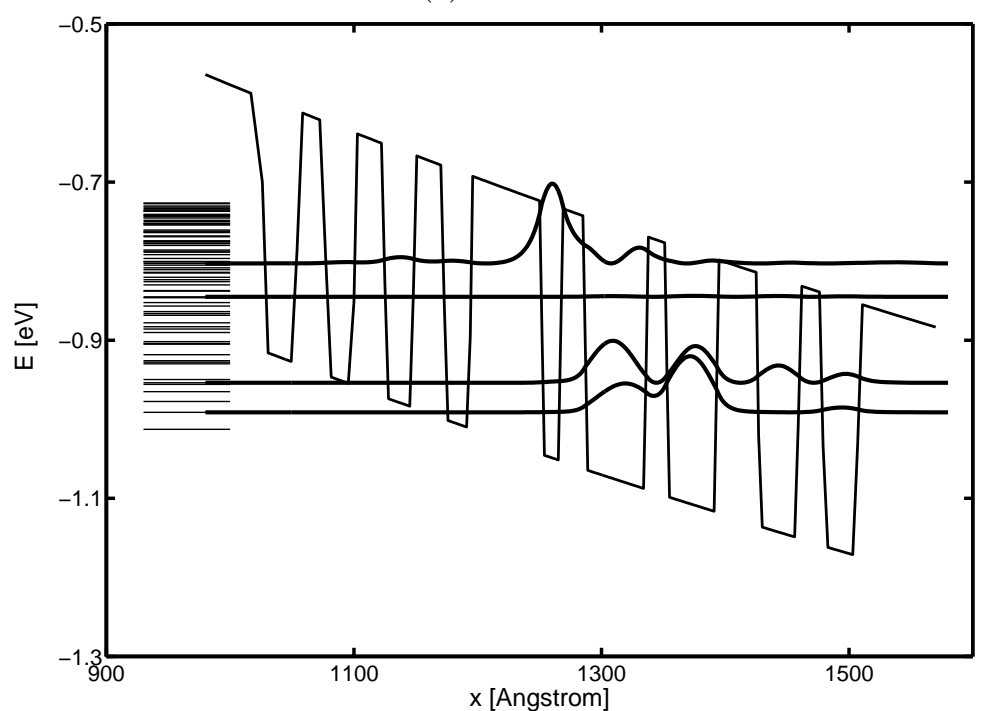

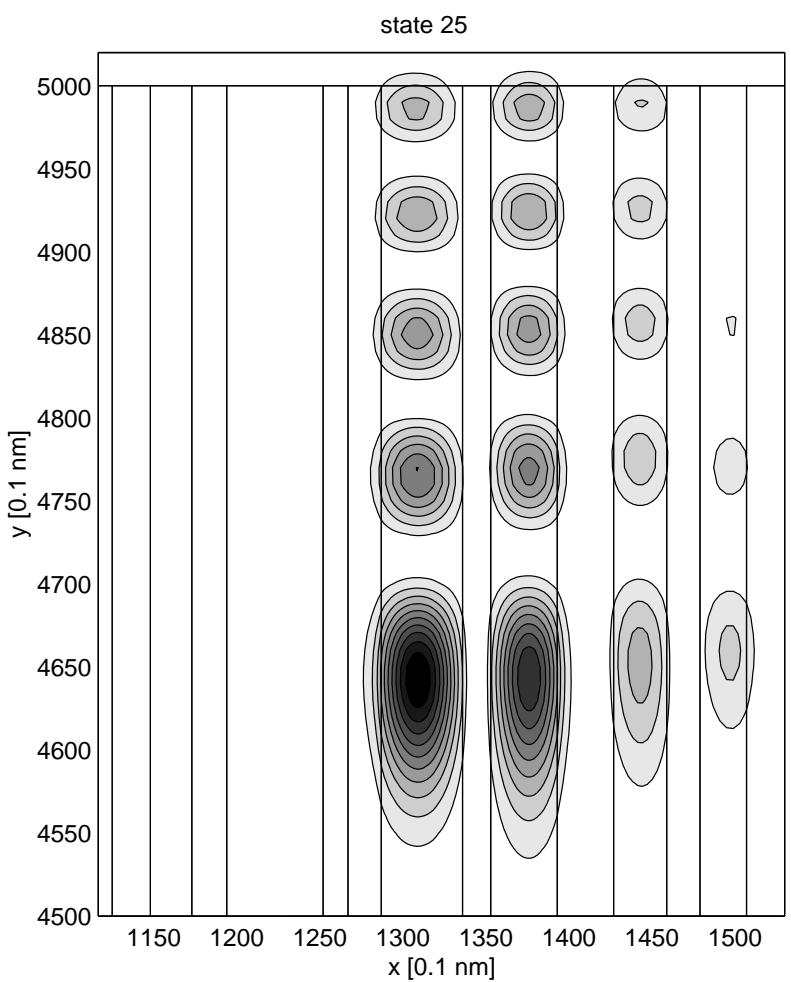

(d)

Fig. 3. Contour plots of the densities $\left|\chi_{\ell}(x, y)\right|^{2}$ for the subbands $\ell=37 \equiv \mathrm{III}$ (a), $\ell=6 \equiv \mathrm{II}$ (b), $\ell=2 \equiv \mathrm{I}$ (c), $\ell=25$ (d) and the Eigenenergies of the first 75 wave functions plus the moduli squared of the four selected wave functions shown in (a) - (d) plotted at their eigenenergies in the potential shown in Fig. 2 cut at $y=4950 \AA$ (e). 

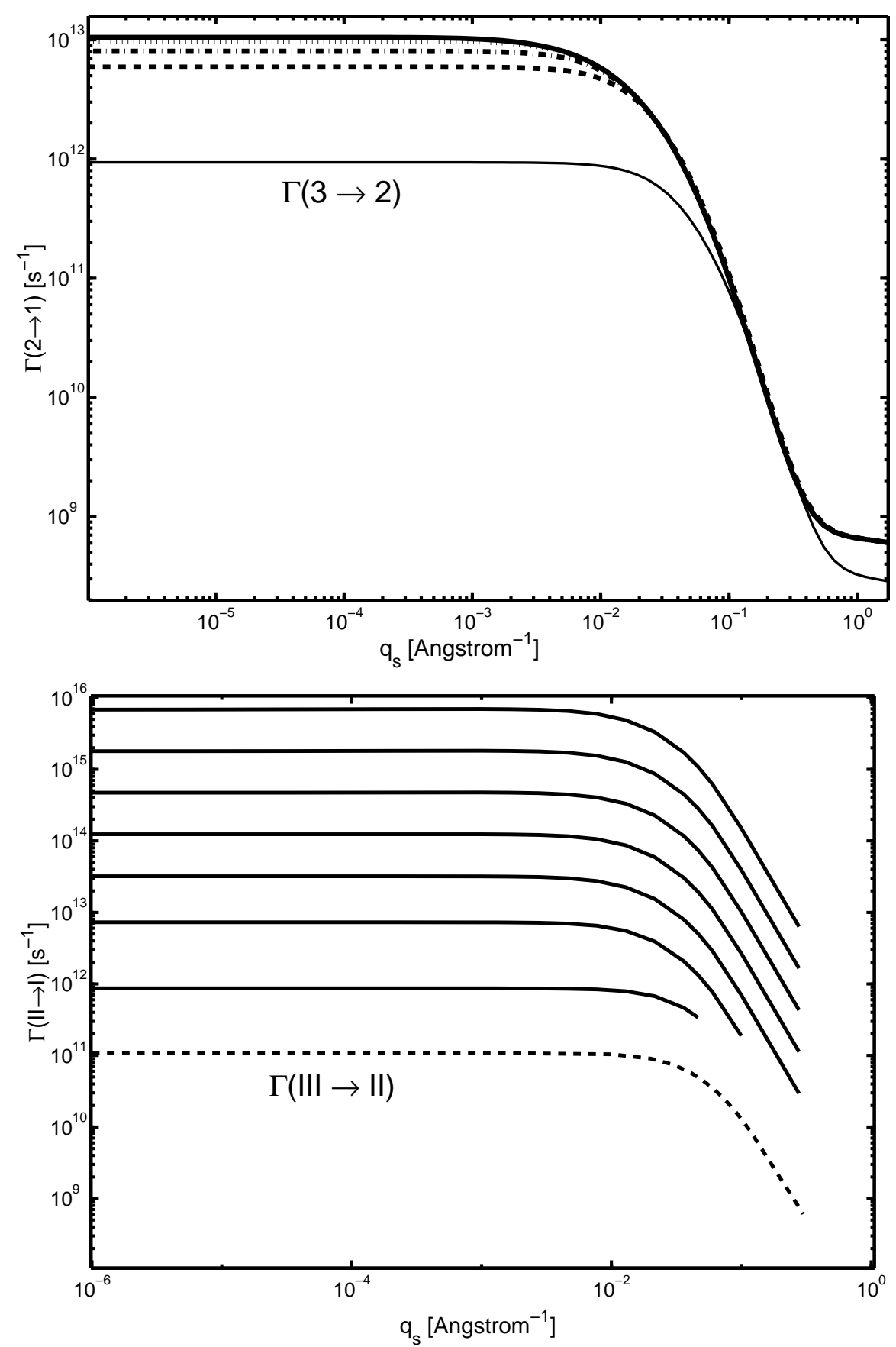

Fig. 4. Dependence of the decay rates $\Gamma_{\mathrm{LO}}(\mathbf{0} 2 \rightarrow 1)$ on the energy difference of states 2 and $1 \Delta E_{2-1}$ for the QCL structure of Ref. Sirtori $\left(\Delta E_{2-1}=50 \mathrm{meV}\right.$ [dashed], $40 \mathrm{meV}$ [dot-dashed], $37 \mathrm{meV}$ [dotted] and $\hbar \omega_{L O}$ [fat solid]) in comparison with the decay rate $\Gamma(3 \rightarrow 2)$ [solid] (a) and $\Gamma_{\mathrm{LO}}(0 I I \rightarrow I)$ for the quantum wire system $\left(\Delta E_{I I I-I I}\right.$ [dashed] and energy separations of $\Delta E_{I I-I}=136.5 ; 43.31292 ; 36.96416 ; 36.53162 ; 36.50215 ; 36.50015 ; 36.50001 \mathrm{meV}$ [solid] with increasing transition rate) analyzed in this work (b). 


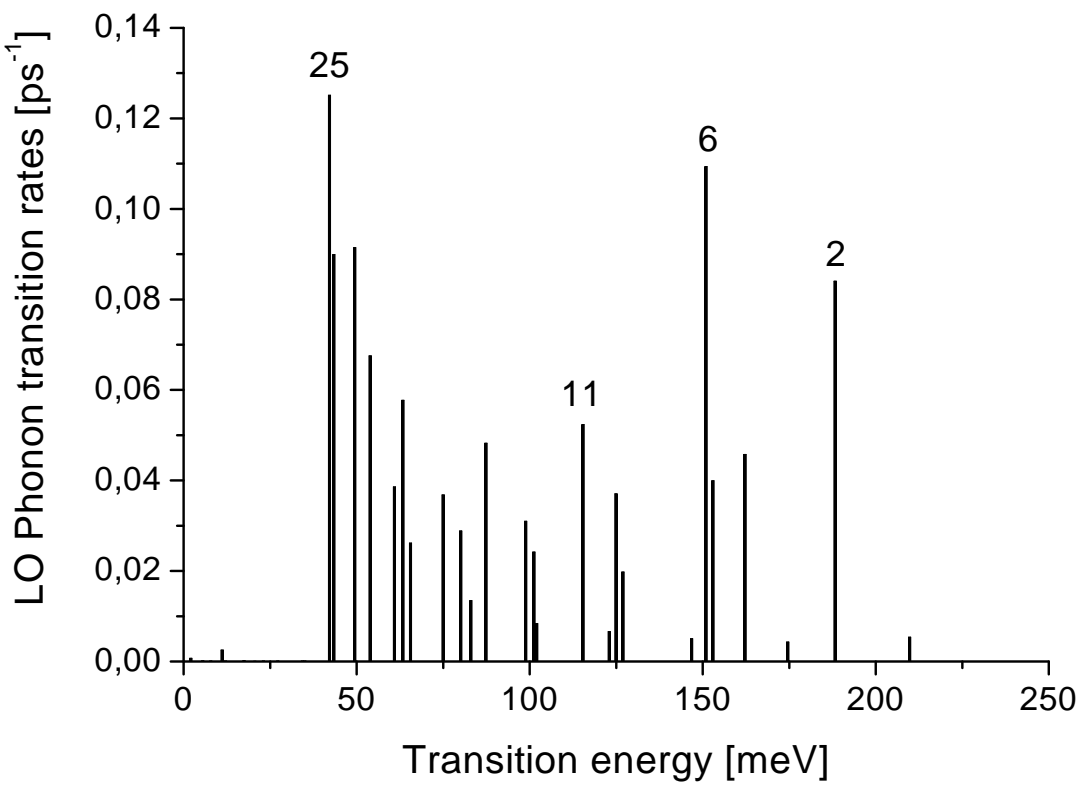

Fig. 5. LO-phonon mediated relaxation rates of the upper level (III) of the active region in the quantum wire system. The height of the vertical bars is proportional to $\Gamma_{\mathrm{LO}}(0 I I I \rightarrow \ell)$, the horizontal position of the bar is at the energy value $E_{\ell}(0)=\epsilon_{\ell}$. 